\title{
Data management at the Oulu cosmic ray station
}

\author{
Stepan Poluianov ${ }^{(1,2}$, Ilya Usoskin ${ }^{1,2}$, Askar Ibragimov ${ }^{(3}$ \\ Correspondence \\ 1'Sodankylä Geophysical Observatory, University of Oulu, Finland, stepan.poluianov@oulu.fi, ilya.usoskin@oulu.fi \\ ${ }^{2}$ Space Physics and Astronomy Research Unit, University of Oulu, Finland \\ ${ }^{3}$ Independent researcher, Helsinki, Finland, askar.ibragimov@gmail.com
}

\section{OPEN ACCESS}

This work is published under the Creative Commons Attribution 4.0 International licence (CC BY 4.0) Please note that individual, appropriately marked parts of the work may be excluded from the licence may be excluded from the licence other copyright condiojs. other copyright conditions. If such thirdparty material is not under the Creative Commons license any copying, editing or public reproduction is only permitted with the prior consent of the respective copy right owner or on the basis of relevant legal authorization regulations.

\section{Keywords}

cosmic rays; neutron monitor; data management

\begin{abstract}
With the recent electronics upgrade of Antarctic neutron monitors (NMs) DOMC and DOMB in 2019, the Oulu cosmic ray station (Sodankylä Geophysical Observatory, Finland) receives a significantly larger amount of data than before. This has led to a need for an important upgrade of the configuration of servers working at the station. The new configuration has three types of servers: a web-server, a datamaster server and data acquisition machines. The web-server provides a user interface for services of the station: the main website, the GLE database and other services. The datamaster is the main server, which stores all data in raw files and a database. Data acquisition machines are computers that directly receive data from the instruments and send the files farther to the datamaster server. This work describes technical details of the cosmic ray station setup providing reliable and secure data acquisition, handling and publication.
\end{abstract}

\section{Introduction}

With new NM DOMC/DOMB, and particularly with much higher data flow caused by their new electronics (see Poluianov, Usoskin and Strauss, this issue), the Oulu cosmic ray station needed an upgrade of its data management system. The changes were aimed to increase the reliability of the system and to raise its capability for new instruments and data.

\section{What is new?}

Briefly, the new improvements in the data management system are:

- Data storage and web-services split into different machines,

- additional acquisition computer for tests at OULU NM,

- new backups to remote storages SGO Jako and CSC Allas,

- new alerting system,

- new code repository and its deployment,

- new wiki-like documentation.

Most of them are described in detail in the following sub-sections. 


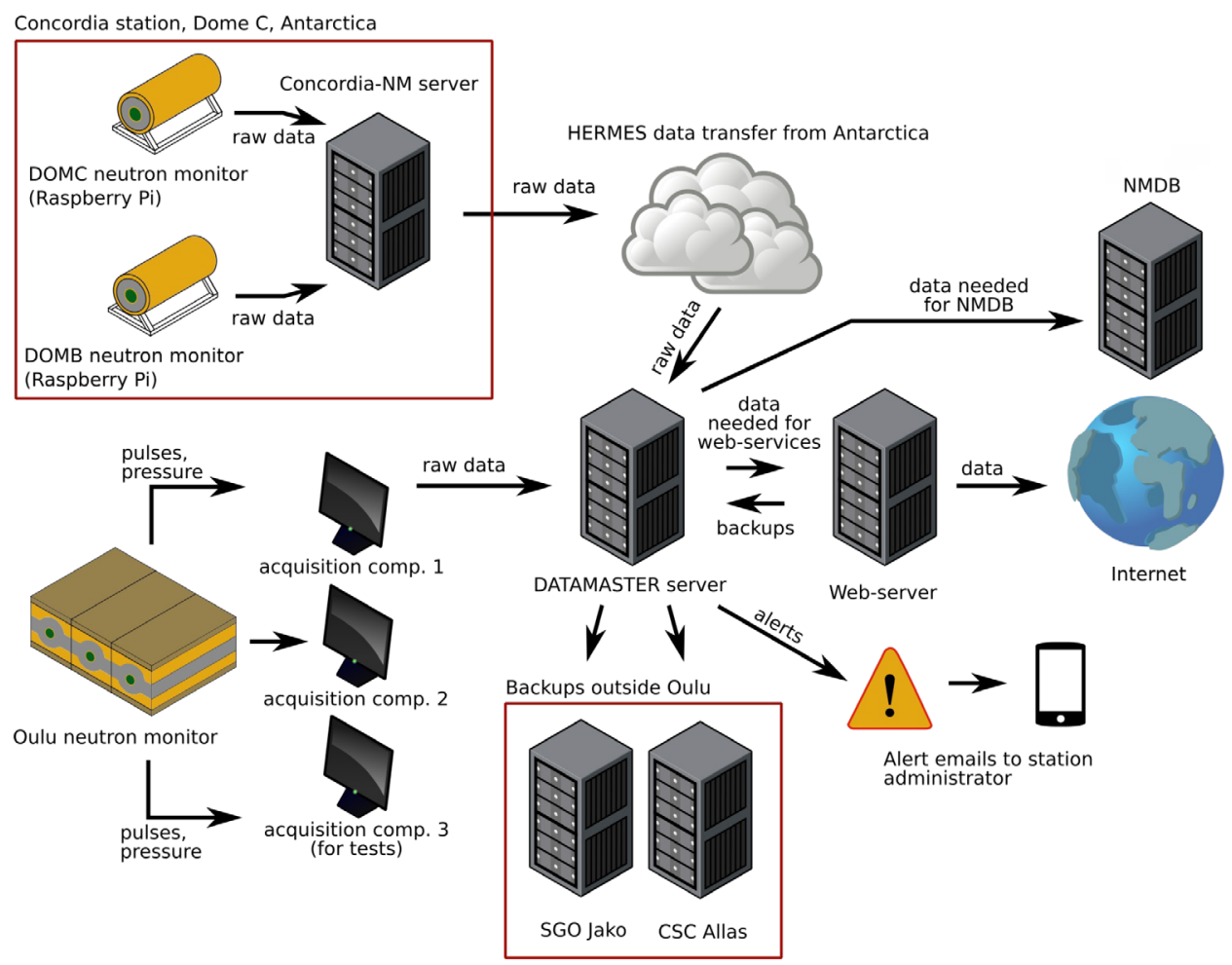

Figure 1: Data flow of the Oulu cosmic ray station.

\subsection{Split data acquisition, storage and web services}

OULU NM has two independent data acquisition chains (TTL-converters, counters, barometers, GPS-receivers, watchdogs, computers) for reliability. It also has an additional acquisition chain for the development of software and tests. Raw data from OULU, as well as from DOMC/DOMB go to a dedicated data-server. That machine has the only purpose to keep original raw files from instruments and a database. It was designed and set up for that particular purpose. For safety reasons, the data-server and acquisition computers are protected by a firewall and are not available from the internet. Access of users to the cosmic ray data is provided via the web-server, which hosts also various station services, e.g., websites http://cosmicrays.oulu.fi, http://gle.oulu.fi (last accessed April 12, 2021).

\subsection{Backup}

All important parts of the system are backed up daily. We follow and even exceed the so-called 3-21 rule (actually, we have more than 3 copies, 2 types of backups, with all copies stored in different locations in Finland). For that purpose, we use software Restic (https://restic.net, last accessed April 12, 2021). We back up not only data but also the code repository, configuration playbooks, documentation and websites.

\subsection{Alerts}

To react fast to possible non-nominal situations with measurements, there are set up alert emails, which go to station administrators. We use the service SendGrid (https://sendgrid.com, last accessed April 12, 2021) for that purpose. 


\subsection{Code repository and deployment}

New instruments and services led to an increase of the amount of written code (more programs, scripts, configuration files, documentation, etc.), which should be also stored properly (safely, with version control and backups). We use Git (https://git-scm.com, last accessed April 12, 2021) with our own repository hosted at the web-server. Handling the code stored in Git adds more convenience and reliability in the development of station services. With more servers at work, automatic configuration and code deployment were established. This also makes the station much less dependent on the experience level of a single person. This function is realized with software Ansible (https:// www.ansible.com, last accessed April 12, 2021).

\subsection{Documentation}

All documentation (logbooks, descriptions and configurations of instruments and servers, instructions) are regularly updated and are available at a protected wiki-like website hosted at the web-server. This website is run with Confluence (https://www.atlassian.com/software/confluence, last accessed April 12 , 2021). The documentation is a "reference book" for the team describing how to work with instruments, servers and data, how to share, update files and so on. This is also an important component of making the station less dependent on a single person.

\section{Data revision policy}

The station has the rule "No modification of raw data" applied to all acquired data. All changes are stored in copies of raw data files labeled as "corrected" and/or in the database (pressure, efficiency, temperature correction coefficients). The original raw files serve as the only "source of truth".

\section{Summary}

The instrumental basis and computer infrastructure of the Oulu cosmic-ray station have been significantly upgraded and expanded during recent years. The list of changes includes separation of data storage and web-services to different machines, a new backup system, new email alerts, Git version control of the code and configurations, comprehensive documentation. The described changes at the Oulu cosmic ray station have significantly increased its reliability of operation and capacity to new data and instruments if they come.

\section{Acknowledgments}

The work was partly supported by FINNARP and Academy of Finland (projects 264378 CRIPA, 304435 CRIPA-X, ReSoLVE and 321882 ESPERA).

\section{References}

Ansible, https://www.ansible.com (last accessed November 23, 2020)

Atlassian, https://www.atlassian.com/software/confluence, (last accessed November 23, 2020)

Git community, https://git-scm.com (last accessed November 23, 2020)

Restic community, https://restic.net (last accessed November 23, 2020)

Twilio SendGrid, https://sendgrid.com (last accessed November 23, 2020) 